\title{
Angular momentum evolution in M67
}

C. Melo

ESO, Casilla 19001, Santiago 19, Chile, cmelo@eso.org

L. Pasquini

ESO, Karl-Schwarzschild-Strasse 2, 85748 Garching bei Mnchen, Germany, lpasquin@eso.org

\section{J.R. De Medeiros}

Departamento de Física Teórica e Experimental - UFRN, Brazil, renan@dfte.ufrn.br

\begin{abstract}
We present nearly 70 new observations for M67 members at the main-sequence (MS), turn-off (TO) and post-turn-off (PTO) in addition to those already published in Melo, Pasquini \& De Medeiros (2001). These additional $v \sin i$ measurements confirm that most of stars are experiencing a strong braking as they pass the turn-off. In contrast to our previous results, some spread in the $v \sin i$ is observed among the TO and PTO stars.
\end{abstract}

\section{Introduction}

Melo, Pasquini, \& De Medeiros (2001, MPD) measured accurate projected rotational velocity $v \sin i$ for $28 \mathrm{M} 67$ members in different evolutionary stages from the main-sequence to the sub-giant branch (SGB). The results from MPD were the following: i) very little spread was observed within individual groups, ii) stars spin-up when they reach the turn-off and iii) the spin-down which follows when the stars evolve off from the MS is better fitted by a $I \Omega^{0.5}=C$ (angular momentum being depleted) rather than a $I \Omega=C$ (angular momentum conservation law).

Here we have increased our M67 sample in order to check whether our previous results still hold. We added nearly 70 new high-resolution spectroscopic observations to those 28 already published in MPD. All observations were carried out with FEROS at the $1.52 \mathrm{~m}$, ESO, Chile. Observations, data reduction and analysis were performed as described in MPD.

\section{Results}

The cumulative distribution of the $v \sin i$ for the four different groups of stars in M67 is shown on the left-hand side of Figure 1. A spin-up compared to the $v \sin i$ distribution of the MS is clearly seen among the TO members. All stars in the MS group have $v \sin i$ less than $5.5 \mathrm{~km} \mathrm{~s}^{-1}$ whereas for the TO stars, $v \sin i$ are mostly between $5.5 \mathrm{~km} \mathrm{~s}^{-1}$ and $8.0 \mathrm{~km} \mathrm{~s}^{-1}$. Just after passing the turn-off the rotation drops, $70 \%$ of the stars in this group have $v \sin i \leq 5.5 \mathrm{~km} \mathrm{~s}^{-1}$, although 

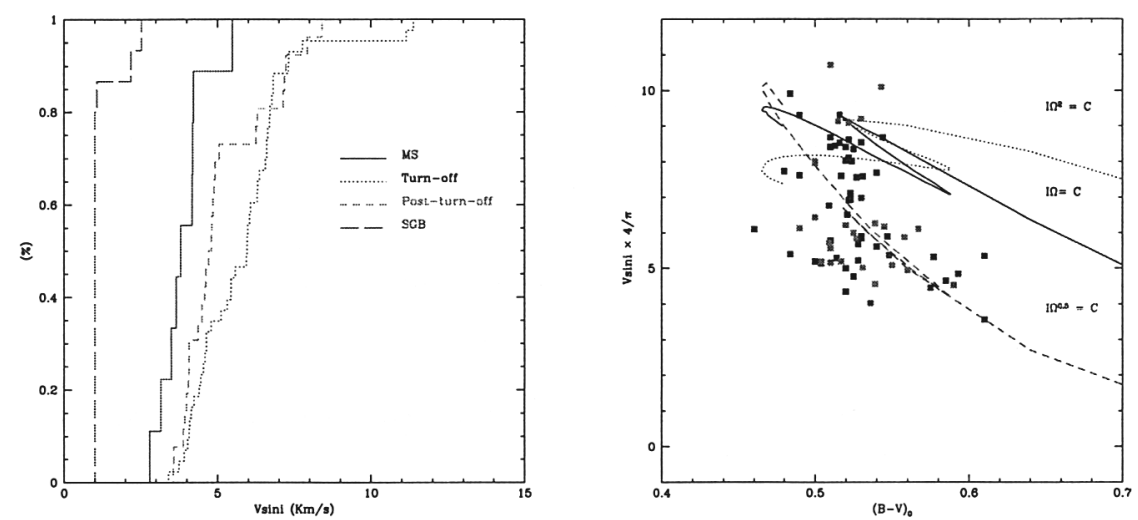

Figure 1. Left. Cumulative distribution for MS, TO, PTO and SGB stars. Right. Rotational velocity as function of $(B-V)_{0}$. MS, TO and PTO stars are represented by triangles, circles and squares, respectively

$30 \%$ of the stars in the PTO group still have a $v \sin i$ comparable to that of the TO. Finally, at the SGB, only upper limits could be found for almost all stars in this group, $76 \%$ have $v \sin i$ less than $1.0 \mathrm{~km} \mathrm{~s}^{-1}$ and the highest $v \sin i$ is less than $3.0 \mathrm{~km} \mathrm{~s}^{-1}$.

On the right-hand side of Figure 1, we compare our $v \sin i$ 's converted into rotational velocities with the theoretical rotational velocity curves derived according three different assumptions for the angular momentum (AM): i) $I \Omega^{2}=$ $C$, AM is transfered from the core; ii) $I \Omega=C$, AM is conserved; iii) $I \Omega^{0.5}=C$ $\mathrm{AM}$ is depleted. Here we see that the rotational drop present in the cumulative distribution as the stars pass the turn-off is better fitted by a $I \Omega^{0.5}=C$. This suggests that in very short time, angular has been highly depleted. The further drop in the rotation as the stars enter in the SGB, follows a AM conservation law (see MPD).

These results rise some interesting questions. Rotation at the MS is relatively uniform which is expected from the angular momentum scenarios at the age of M67 (e.g., Bouvier et al. 1997). However, is not clear why there are stars in the turn-off which don't spin-up and why some stars don't spin-down after passing it. Are they experiencing any angular momentum transfer from the interior? What would be the impact on the light element abundances predicted by theoretical models where rotation has been incorporated (e.g. Chaname et al. 2003)? Can the rotational distribution of M67 be reproduced by the current scenarios? These issues will explored in detail in a future paper.

\section{References}

Bouvier, J., Forestini, Allain, S. 1997,, A\&A326, 1023

Chaname, J., Pinsonneault, M., Terndrup D. 2003, this volume

Melo, C., Pasquini, L., De Medeiros, J.R. 2001, A\&A375, 851 